\title{
Semiautomated determination of minimum bactericidal concentrations of antibiotics
}

\author{
F O'GRADY, BARBARA MURPHY, NJ PEARSON \\ From the Department of Microbiology and Public Health Laboratory, University Hospital, Queen's Medical \\ Centre, Nottingham NG72UH
}

SUMMARY Using a newly devised 50-channel photometer which records the opacity of growing bacterial cultures, it was shown that the time taken by cultures diluted $1 / 1000$ in fresh broth to reach $50 \%$ of the opacity of a fully grown culture was inversely related to the concentration of organisms in the original culture. This relation was used to determine the numbers of survivors after exposure to benzylpenicillin and gentamicin alone and in combination. The procedure is commended as a labour-saving and potentially rapid method of obtaining comprehensive information on the bactericidal action and interaction of antibiotics.

Several fully automatic methods have been developed for determining susceptibility of bacteria to antibiotics, some of which measure the minimum inhibitory concentration. Many methods use opacity measurements of growth of bacteria in the presence of antibiotics, but automatic reading of multipoint inoculated plates containing antibiotics is also possible. ${ }^{1}$ Where it is necessary to determine the bactericidal activity of agents alone or in combination, manual methods are employed which require either formal determination of the numbers of surviving bacteria after various intervals of exposure to the antibiotic or, for the purpose of guiding therapy, determine simply the minimum concentration of agents or mixture from which acceptably few survivors can be recovered after overnight incubation.

The first of these methods is extremely labourintensive-unacceptably so in the diagnostic laboratory if several agents have to be tested in combination-and the second method provides minimal information about antibiotic interactions. Because of overnight incubation, the methods are slow, the period of exposure of bacteria to antibiotic is much longer than the interval between therapeutic doses and counts based on transfer from fluid to solid media may underestimate the number of survivors as a result of mechanical damage to antibiotic-injured cells or failure of cells to adapt to new cultural conditions.

In an attempt to overcome these difficulties we

Accepted for publication 22 September 1982 have examined the possibility that the time taken for regrowth to occur when antibiotic-treated cultures are diluted with fresh medium could be used as a measure of the number of survivors. For many years we have used regrowth as a measure of the number of survivors in mechanical models simulating conditions of treatment of bacterial cystitis ${ }^{2}$ and more recently we have shown that the time taken for detectable growth to reappear in diluted cultures is systematically related to the number of organisms originally present. $^{3}$ We have now applied this method to cultures exposed to bactericidal agents in the expectation that regrowth from such cultures after the addition of sufficient fresh medium to dilute residual antibiotic well below inhibitory concentrations will provide a reproducible measure of the number of survivors.

\section{Material and methods}

\section{CAROUSEL DEVICE}

To measure the opacity of growing cultures we have used the multichannel carousel device previously $\omega$ described. ${ }^{3}$ Briefly, the device consists of a black anodised aluminium drum drilled close to its edge to accommodate fifty $10 \mathrm{~mm} \times 50 \mathrm{~mm}$ test tubes. At positions corresponding with the tubes vertical slots are cut in the drum which rotates at 10 revolutions per hour in such a way that each tube in turn passes between a stabilised light source and a photocell. As each tube passes, the light falls on the photocell, rises to a peak and then declines. The peak reading is stored on a floppy disc controlled by a Commodore 
8032 microcomputer for later access. The carousel is housed in an incubator at $37^{\circ} \mathrm{C}$ and when the tubes contain broth inoculated with bacteria, the opacity increases as growth occurs and the peak signal declines, an almost linear inverse relation being obtained between opacity and peak signal. At the end of a preset period of incubation, the growth curves and the time taken by the diluted cultures to reach $50 \%$ of the opacity of a fully grown culture can be generated by the microcomputer and displayed graphically by a programmable plotter (Watanabe WX4671).

\section{CALIBRATION}

Overnight cultures in Eugon broth were diluted by tenfold steps and a $1 / 1000$ dilution of each made by further addition of Eugon broth. The tubes were loaded into the carousel and incubated overnight. The growth curves were generated and the time taken for growth in each tube to reach $50 \%$ of the opacity of a fully grown culture was plotted against the original concentration of organisms.

\section{BACTERICIDAL ACTIVITY}

Doubling dilutions of appropriate concentrations of penicillin or gentamicin were made in Eugon broth and $1 \mathrm{ml}$ volumes inoculated with one drop of a 1/100 dilution of overnight culture. In some experiments, in order to determine the time course of the bactericidal effect, aliquots were removed at hourly intervals and diluted 1/1000 in Eugon broth. In other experiments, the effects of different concentrations of the antibiotics were similarly determined after $6 \mathrm{~h}$ incubation (when bactericidal activity was expected to be nearly complete) by preparing $1 / 1000$ dilutions which were then loaded into the carousel and incubated overnight. The times taken to reach $50 \%$ opacity (or, using the relation between number of organisms and time to regrow generated by the calibration experiments, the numbers of survivors) was computer-plotted against time of exposure or against antibiotic concentration.

\section{BACTERICIDAL SYNERGY}

Two sets of tubes were prepared containing doubling dilutions from 16-1 mg gentamicin per litre and 2-0.1 mg penicillin per litre from which a matrix of 36 tubes containing $2 \mathrm{ml}$ volumes of a control and all concentrations of each agent alone and in combination was prepared. Each tube was inoculated with one drop of $1 / 100$ overnight culture. One set of tubes was incubated overnight and $0.01 \mathrm{ml}$ volumes then transferred to $1 / 4$ blood agar plates by means of a Tarr's loop in a conventional measure of bactericidal activity. The plates were incubated overnight and growth recorded as + (about 1000 colonies); \pm (<1000 colonies); - (no growth). The second set of tubes was incubated for six hours when dilutions of $1 / 1000$ were prepared in Eugon broth, dispensed into carousel tubes and incubated overnight. The following morning the times taken to reach $50 \%$ opacity were computer-plotted against gentamicin concentrations as the family of curves representing the series of penicillin concentrations or against penicillin concentrations as the family of curves representing the series of gentamicin concentrations.

\section{Results and discussion}

Figure 1 shows the curves generated from serial tenfold dilutions of an overnight culture of a strain of Streptococcus faecalis and Fig. .2 the computerplotted relation between the original concentration of organisms and the time for the $1 / 1000$ culture dilutions to reach $50 \%$ of the opacity of fully grown cultures of Strep faecalis (six experiments) and $E$ coli (five experiments). Using the demonstrated relation between numbers of Strep faecalis and regrowth times

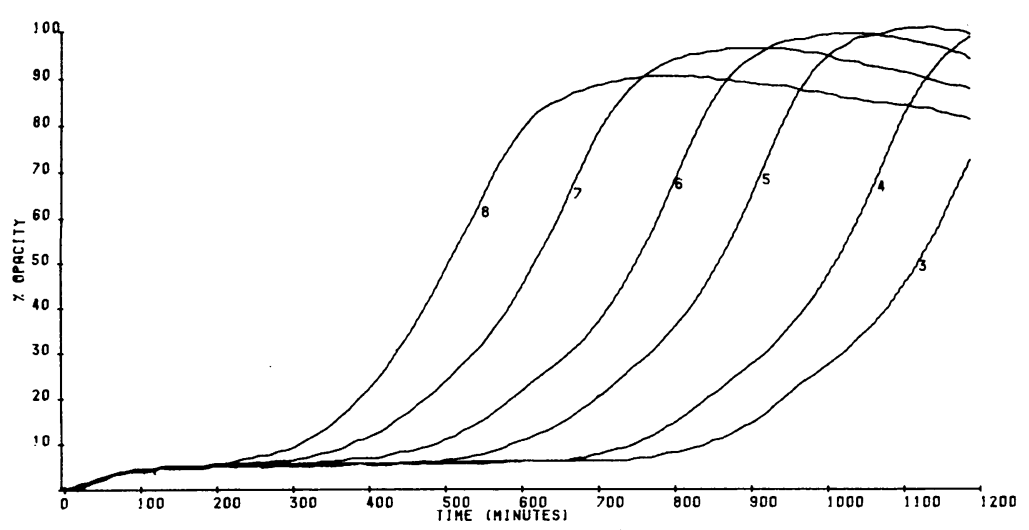

Fig. 1 Typical examples of growth curves of Strep faecalis in Eugon broth generated by the method described showing the relation between time taken for growth to occur and the original concentration of organisms $\left(10^{8}-10^{3} / \mathrm{ml}\right)$. 


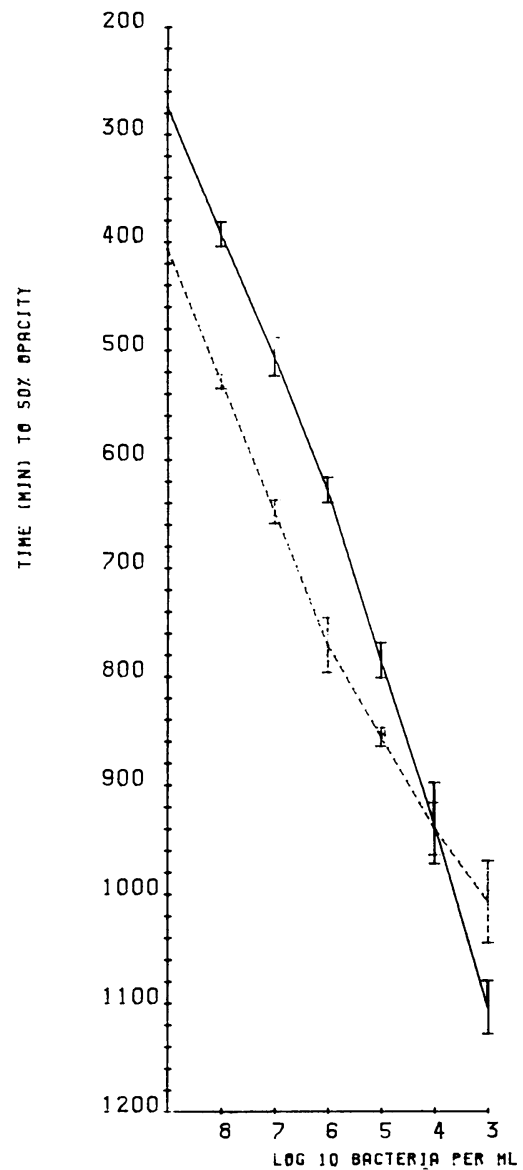

Fig. 2 Correlation between original concentration of Strep faecalis (six separate calibrations) and E coli (five separate calibrations) and time taken for the opacity to reach $50 \%$ of that of a fully grown culture. Bars show the range of values obtained.

Fig. 4 Numbers of survivors after $6 \mathrm{~h}$ exposure to various concentrations of benzylpenicillin and gentamicin.

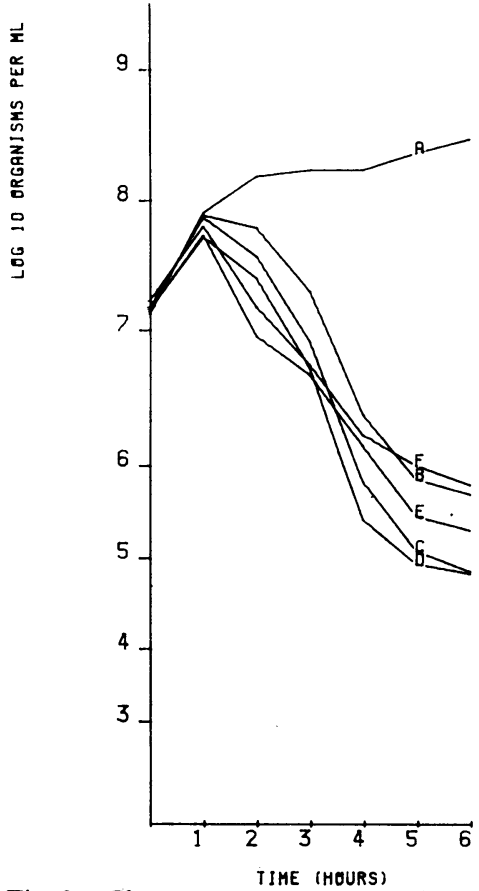

Fig. 3 Change in number of survivors with time during exposure to

benzylpenicillin ( $\mathrm{mg} / \mathrm{l})(A) 0.25(B)$

$0 \cdot 5$ (C) 1 (D) 2 (E) 4 (F) 8 .

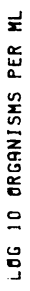

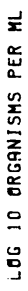

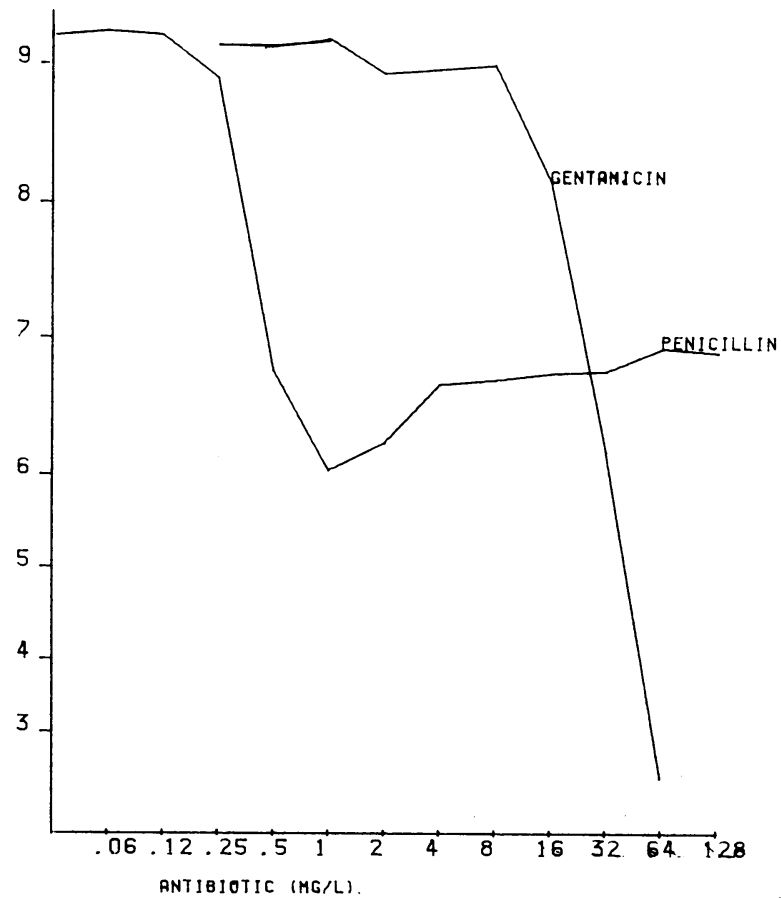




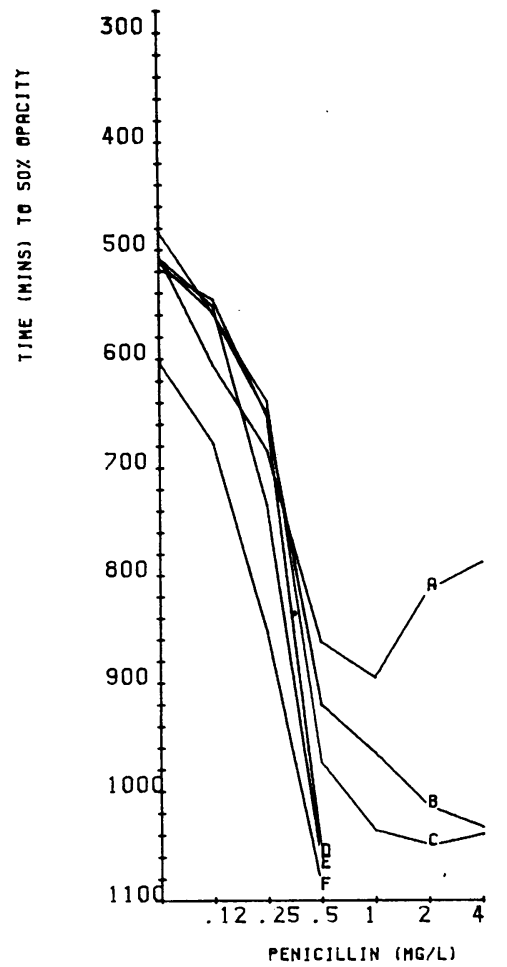

Fig. 5 Numbers of survivors after 6 h exposure to various concentrations of benzylpenicillin in the absence $(A)$ or the presence of gentamicin $(\mathrm{mg} / \mathrm{l})(B) 0.5(C) 1(D) 2(E) 4(F) 8$.

(Fig. 2), Fig. 3 shows the computer plot of the decline in the number of survivors exposed to various concentrations of penicillin: there is little further fall in the number of "persisters" after about a five-hour exposure. Figure 4 clearly shows the contrast in bactericidal activity after six-hour exposure to increasing concentrations of penicillin or gentamicin. With penicillin there is an optimum bactericidal concentration (the well-known Eagle effect) above which there is no further reduction in the number of "persisters." With gentamicin, as the concentration is increased above the minimum effective level (high in Eugon broth), there is a precipitate decline in the number of survivors. Striking synergy between penicillin and gentamicin was shown by the marked reduction in survivors, as indicated by prolonged regrowth times, in the presence of the two agents (Figs. 5 and 6) where it is particularly noteworthy that very little extra benefit accrued from increasing either penicillin or gentamicin above the lowest effective concentrations.

The results obtained by a conventional "chessboard" titration of combined bactericidal activity (which customarily involves two periods of

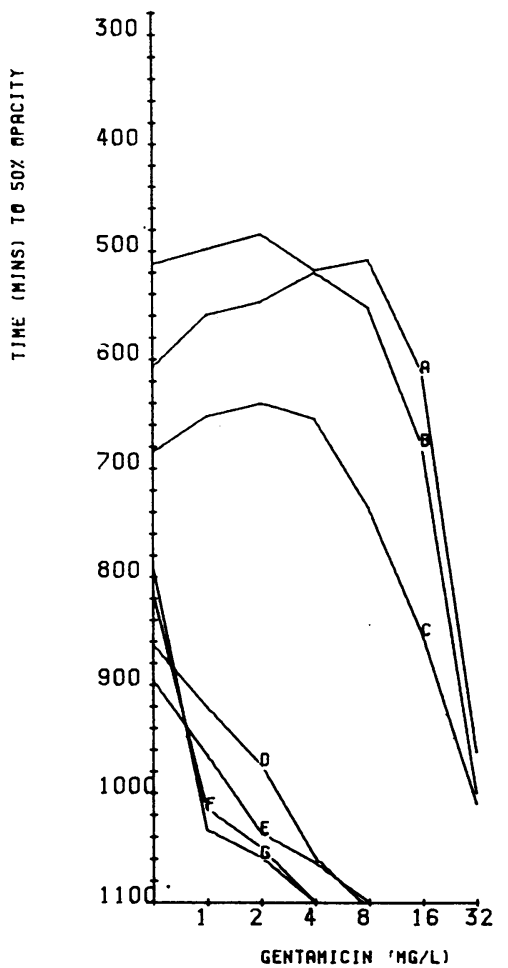

Fig. 6 Number of survivors after $6 h$ exposure to various concentrations of gentamicin in the absence $(A)$ or presence of benzylpenicillin $(\mathrm{mg} / \mathrm{l})(B) 0.12$ (C) 0.25 (D) $0.5(E) 1$ (F) 2 (G) 4.

Comparative results of chessboard titration by conventional and semiautomated methods

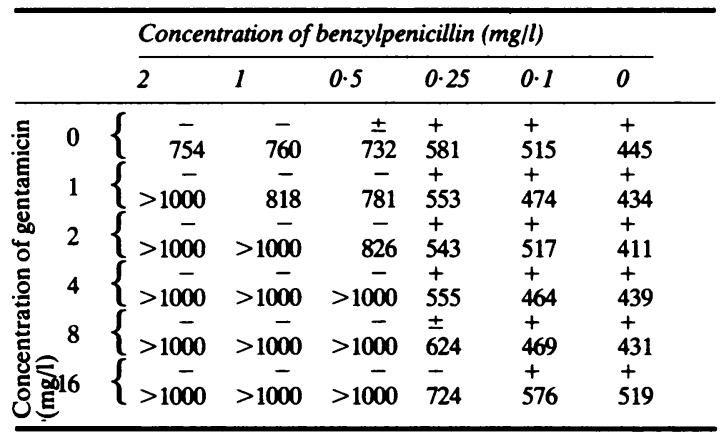

Assessment of numbers of survivors $(+; \pm ;-)$ in conventional titration of bactericidal activity and corresponding regrowth times ( $\mathrm{min}$ ) in semiautomated assay of a strain of Strep faecalis exposed to the antibiotic concentrations.

overnight incubation) and the semiautomated method have been compared for strains of streptococci isolated from patients with infective endocarditis. A typical comparison for a strain of 
Strep faecalis is shown in the Table. Growth comparable to that of controls in the conventional method corresponded with regrowth times in the semiautomated method up to about $600 \mathrm{~min}$. The two tubes showing reduced numbers of survivors in the conventional titration showed regrowth times of 624 and $732 \mathrm{~min}$. Tubes showing longer regrowth times all showed no growth by the conventional method.

It appears from the calibration experiments that reproducible relations can be shown between numbers of organisms and regrowth times in the conditions described. Such regrowth times can be used as a measure of the number of organisms capable of regrowth after exposure to antibiotics and results obtained in this way have corresponded with those obtained by a conventional measure of bactericidal activity.

The patterns of the bactericidal activity of benzylpenicillin and gentamicin are entirely consonant with those obtained by conventional methods and the

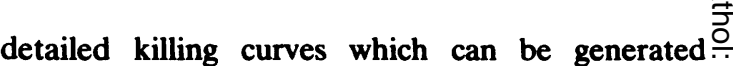
relatively effortlessly by this method should be $\vec{\Rightarrow}$ helpful in elucidating the nature and magnitude of bactericidal action and interaction.

\section{References}

' McDonald T, Pearson N, Johnson F, O'Grady FW. Automated reading of the multipoint inoculated antibiotic sensitivity test. कs J Med Eng Technol 1981;5:243-5.

${ }^{2}$ Greenwood D, O'Grady F. An in vitro model of the urinary. bladder. J Antimicrob Chemother 1978;4:113-20.

${ }^{3}$ O'Grady F, Pearson NJ, Ridout P, Murphy B, McDonald T, O Johnson F. Automated photometry for detecting bacteriuria. In: Tilton RC, ed. Rapid methods and automation in $\mathrm{O}$ microbiology. American Society for Microbiology, 1982:166- W 70 .

Requests for reprints to: Professor F O'Grady, Department 음 of Microbiology and Public Health Laboratory, University Hospital, Queen's Medical Centre, Nottingham NG7 2UH, T England. 